\title{
Collecting Experience on the Systematic Development of CBR Applications using the INRECA Methodology
}

\author{
Ralph Bergmann ${ }^{1}$, Sean Breen ${ }^{2}$, Emmanuelle Fayol ${ }^{3}$, Mehmet Göker ${ }^{4}$, \\ Michel Manago ${ }^{3}$, Sascha Schmitt ${ }^{1}$, Jürgen Schumacher ${ }^{1}$, Armin Stahl ${ }^{1}$, \\ Stefan Wess ${ }^{5}, \&$ Wolfgang Wilke ${ }^{1}$ \\ ${ }^{1}$ University of Kaiserslautern, Centre for Learning Systems and Applications (LSA), PO- \\ Box 3049, D-67653 Kaiserslautern, Germany \\ e-mail: \{bergmann|jschuma|sschmitt|stahl|wilke\}@informatik.uni-kl.de \\ 2 Interactive Multimedia Systems (IMS), Clara House, Glenageary, Co. Dublin, Ireland \\ e-mail: sbreen@imsgrp.com \\ 3 AcknoSoft, 58 a, rue du Dessous des Berges, 75013 Paris, France \\ e-mail: manago@ibpc.fr \\ ${ }^{4}$ Daimler Benz AG - Forschung und Technik, Evolutionäre Systeme (F3S/E), Postfach \\ 2360, D-89013 Ulm \\ e-mail: mehmet_goker@ep.mbag.sifi.daimlerbenz.com \\ 5 TECINNO GmbH, Sauerwiesen 2, D-67661 Kaiserslautern, Germany \\ e-mail: wess@tecmath.de \\ www.inreca.com
}

\begin{abstract}
This paper presents an overview of the INRECA methodology for building and maintaining CBR applications. This methodology supports the collection and reuse of experience on the systematic development of CBR applications. It is based on the experience factory and the software process modeling approach from software engineering. CBR development experience is documented using software process models and stored in different levels of generality in a three-layered experience base. Up to now, experience from 9 industrial projects enacted by all INRECA II partners has been collected.
\end{abstract}

\section{Introduction}

Today, there are already a few companies which are specialized in developing CBR applications. Their problem is that they mostly develop their applications in an ad-hoc manner: They do not have guidelines or methods which could help their developers in performing a new project and there are no ways to preserve experience made in performed projects for future use. This can cause serious problems when members of the staff leave, taking their experience with them, and new staff has to be trained. The result is an inefficient or ineffective system development, which cannot be sustained by contemporary organizations. From these problems, the need for a methodology to 
support the development and maintenance of CBR applications arson a few years ago and several approaches in that direction have been proposed (see [3] for an overview). A methodology describes the development of a software system using a systematic and disciplined approach. It gives guidelines about the activities that need to be performed in order to successfully develop a certain kind of product, e.g., any kind of software system, as in our case, a CBR application. A methodology shall use a welldefined terminology, which makes it also possible to collect experiences made in past projects in a structured and reusable way to improve future projects. One of the main driving forces behind the development and the use of a methodology relates to the need for quality in both the products and processes of the development of computerbased systems. The use of an appropriate methodology will provide significant quantifiable benefits in terms of productivity (e.g., reduce the risk of wasted efforts), quality (e.g., inclusion of quality deliverables), and communication (a reference for both formal and informal communication between members of the development team and between the developer and the client) and it will provide a solid base for management decision making (e.g., planning, resource allocation, and monitoring).

This paper describes the INRECA ${ }^{1}$ methodology approach which is based on two relatively new areas in software engineering (SE): experience factory [2] and software process modeling [6]. We developed a methodology based on recent SE techniques which is enriched by up-to-date experience on building and maintaining CBR applications. This CBR experience was identified by analyzing several successful industrial applications developed by the industrial partners of the INRECA-II ${ }^{1}$ consortium.

\section{The INRECA Methodology Approach}

Our approach to a CBR development methodology is itself very "CBR-like". In a nutshell, it captures previous experience from CBR development and stores it in a socalled experience base (a term from the experience factory approach). The entities being stored in the experience base are software process models, or fragments of it such as processes, products, or methods. The experience base is organized on three levels of abstraction: a common generic level at the top, a cookbook-level in the middle, and a specific project level at the bottom.

\footnotetext{
${ }^{1}$ Funding for this work has been provided by the Commission of the European Union (INRECA-II: Information and Knowledge Reengineering for Reasoning from Cases; Esprit contract no. 22196) to which the authors are greatly indebted. The partners of INRECA-II are AcknoSoft (prime contractor, France), Daimler Benz (Germany), TECINNO (Germany), Interactive Multimedia Systems (Ireland), and the University of Kaiserslautern (Germany). See www.inreca.com for details.
} 


\subsection{Experience Factory}

The experience factory idea is motivated by the observation that any successful business requires a combination of technical and managerial solutions which includes a well-defined set of product needs to satisfy the customer, assist the developer in accomplishing those needs and create competencies for future business; a welldefined set of processes to accomplish what needs to be accomplished, to control development, and to improve overall business; a closed-loop process that supports learning and feedback. The key technologies for supporting these requirements include: modeling, measurement, the reuse of processes, products and other forms of knowledge relevant to the (software) business. An experience factory is a logical and/or physical organization that supports project developments by analyzing and synthesizing all kinds of experience, acting as a repository for such experience, and supplying that experience to various projects on demand (see Figure 1). An experience factory packages experience by building informal, formal or schematized models and measures of various software processes, products, and other forms of knowledge via people, documents, and automated support. The main product of an experience factory is an experience base. The content and the structure of an experience base vary based upon the kind of experience clustered in the base.

The relationship between CBR and the experience factory approach is discussed in some detail by Althoff \& Wilke [1]

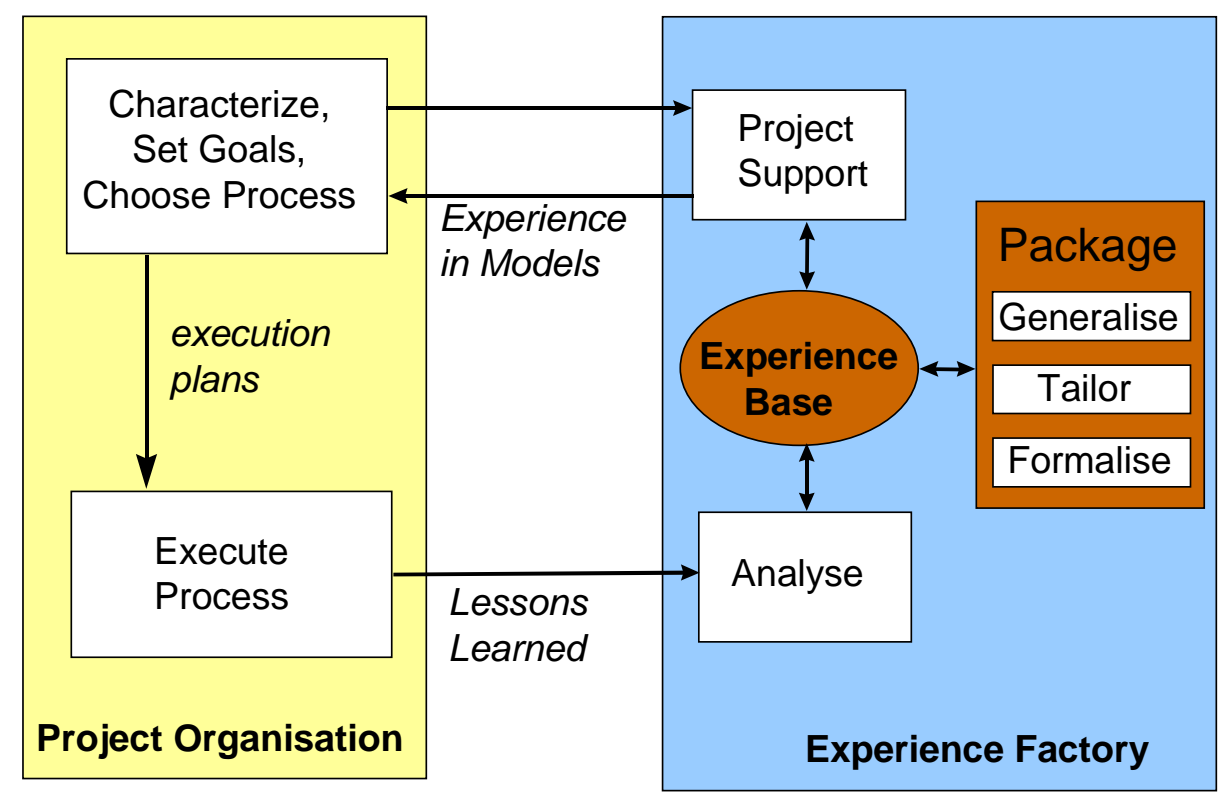

Fig. 1. The Experience Factory Approach [2]. 


\subsection{Software Process Models}

Software process modeling [6] is an approach that is highly important in the context of the experience factory approach. Software process models describe the engineering of a product, e.g., the software that has to be produced. Unlike early approaches in $\mathrm{SE}$, the software development is not considered to follow a single fixed process model with a closed set of predefined steps. A tailored process model particularly suited for the current project must be developed in advance. Software process models include technical SE processes (like requirements engineering, design of the system to be built, coding, etc.), managerial SE processes (like management of product related documentation, project management, quality assurance, etc.), and organizational processes (covering those parts of the business process in which the software system will be embedded and that need to be changed in order to make best use of the new software system). From time to time, such a model has to be refined or changed during the execution of the project if the real world software development process and the model do not match any longer.

Several representation formalisms for process models have been already developed. Although the particular names that are used vary from one representation to another, all representations have a notation of processes, methods, products, goals, and resources. A process is a single step that has to be carried out in a software development project. Each process has a defined goal and it consumes, produces, or modifies certain products. Usually, the goal of a process is to create or modify the products. Products include the executable software system as well as the documentation like design documents or user manuals. For enacting a process, there can be several alternative methods that describe how to actually enact the process. When the process is enacted, an appropriate method must be chosen. We distinguish between simple and complex methods. A simple method can be a textual description like a guideline of what has to be done to reach the goal of the process. A complex method decomposes a process into a set of sub-processes that exchange certain byproducts in the course of achieving the goal of the main process. For a detailed description of the software process modeling approach used in the INRECA-II methodology see [4].

In the INRECA-II methodology, software process models are used to represent the CBR development experience that is stored in the experience base. Software processes being represented can be either very abstract, i.e., they can just represent some very coarse development steps such as: domain model definition, similarity measure definition, case acquisition. But they can also be very detailed and specific for a particular project, such as: analyze data from Analog Device Inc. operational amplifier (OpAmp) product database, select relevant OpAmp specification parameters, etc. The software process modeling approach allows to construct such a hierarchically organized set of process models. Abstract processes can be described by complex methods which are themselves a set of more detailed processes. We make use of this property to structure the experience base. 


\subsection{The Structure of the Experience Base}

The experience base is organized on three levels of abstraction: a common generic level at the top, a cookbook-level in the middle, and a specific project level at the bottom (see Figure 2).

Common Generic Level. At this level, processes, products, and methods are collected that are common for a large spectrum of different CBR applications. These descriptions are the basic building blocks of the methodology. The documented processes usually appear during the development of most CBR applications. The documented methods are very general and widely applicable and give general guidance of how the respective processes can be enacted. At this common level, processes are not necessarily connected to a complete product flow that describes the development of a complete CBR application. They can be isolated entities that can be combined in the context of a particular application or application class.

Cookbook-Level: Experience Modules. At this level, processes, products, and methods are tailored for a particular class of applications (e.g., help desk, technical maintenance, product catalog). For each application class, the cookbook-level contains an experience module. Such an module is a kind of recipe describing how an application of that kind should be developed and/or maintained. Thereby, the items (processes, methods, and products) contained in such a module provide specific guidance for the development of a CBR application of this application class. Usually, these items are more concrete versions of items described at the common generic level. Unlike processes at the common generic level, all processes which are relevant for an application class are connected and build a product flow from which a specific project plan can be developed.

Specific Project Level. The specific project level describes experience in the context of a single particular project that had already been carried out in the past. It contains project specific information such as the particular processes that were carried out, the effort that was spent for these processes, the products (e.g. domain model) that have been produced and methods that have been selected to actually perform the processes and people that had been involved in executing the particular processes. 


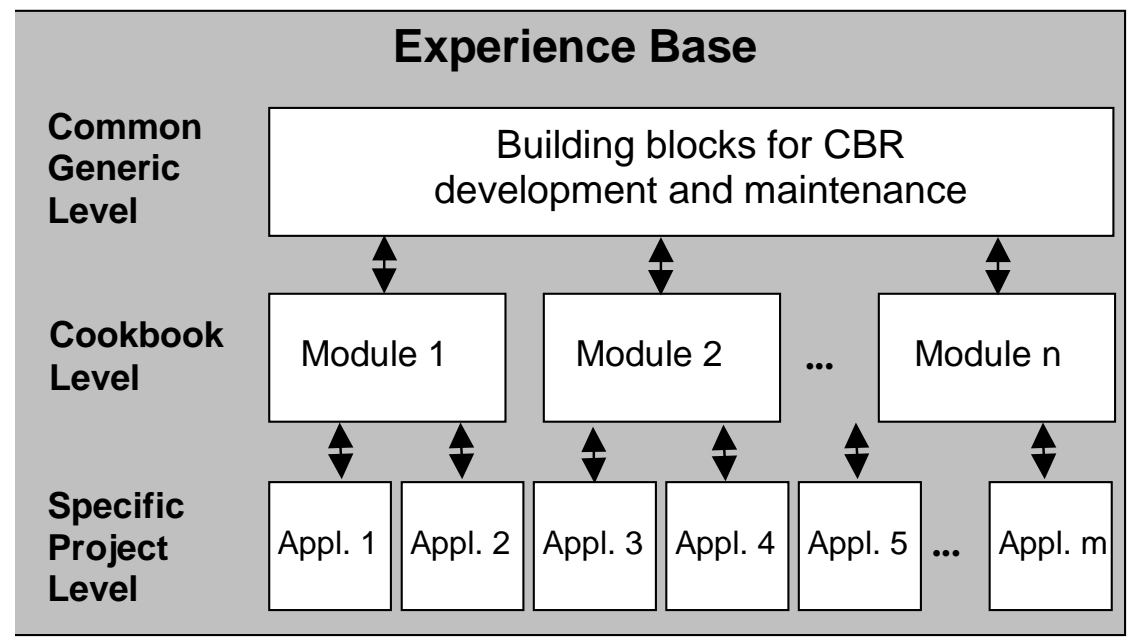

Fig. 2. Structure of the Experience Base.

\subsection{Documentation of the Experience Base}

Processes, products, methods, agents, and tools being stored in the experience base are documented using a set of different types of sheets. A sheet is a particular form that is designed to document one of the items. It contains several predefined fields to be filled as well as links to other sheets (see example in the Appendix). We have developed four types of sheets (for products, processes, simple methods, and complex methods) for documenting generic processes that occur on the top and the middle layer of the experience base and six types of sheets (four sheets for products, processes, simple methods, and complex methods, and two additional sheets for tool and agent descriptions) for documenting specific processes for the specific project level of the experience base. Figure 3 shows the four generic description sheets. One kind of sheet is used to describe generic processes. Generic process sheets contain references to the respective input, output, and modified products of the process. Each product is documented by a separate generic product description sheet. Each process description sheet also contains links to one or several generic methods. A generic method can either be a generic simple method (which is elementary and does not contain any references to other description sheets) or it can be a generic complex method. Such a generic complex method connects several sub-processes (each of which is again documented as a separate generic process description) which may exchange some by-products (documented as separate generic product descriptions). 
As part of the INRECA-II project, a particular methodology tool was implemented which supports the management of the experience base and the different modules it consists of. It supports the filling of the different sheets, checks consistency, and creates the required links. It exports the experience base as an HTML network in which each sheet becomes a separate HTML page that includes links to the related pages. Therefore, it is possible to investigate the experience base via Intranet/Internet using a standard Web browser.

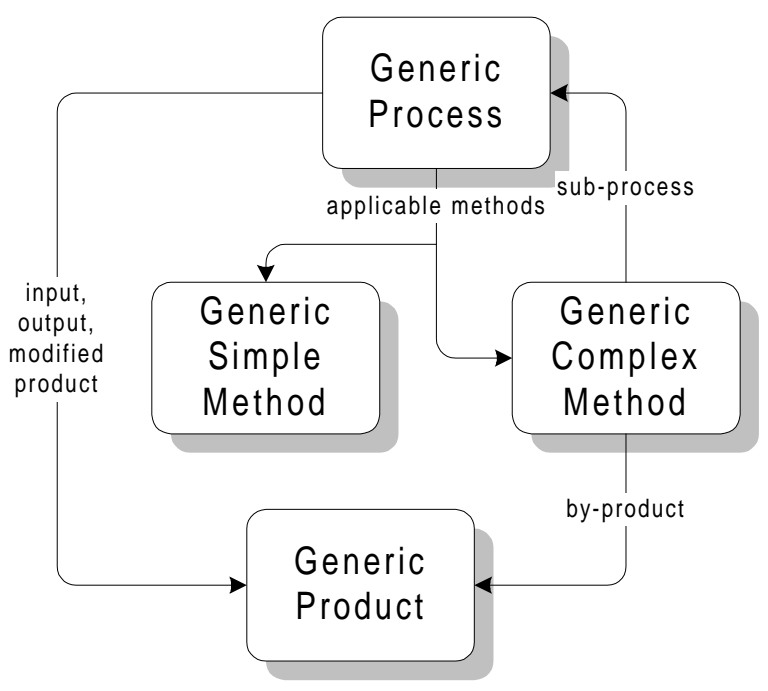

Fig. 3. Overview of generic description sheets.

\subsection{Using and Maintaining the Experience Base}

When a new CBR project is being planned, the relevant experience from the experience base must be selected and reused. The experience modules of the cookbook-level are particularly useful for building a new application that directly falls into one of the covered application classes. We consider the experience modules to be the most valuable knowledge of the methodology. Therefore, we suggest to start the "retrieval" by investigating the cookbook-level and only using the common generic level as fall-back. Furthermore, it is important to maintain the experience base, i.e., to make sure that new experience is entered if required. For using and maintaining the experience base we propose the following procedure:

\footnotetext{
${ }^{2}$ Up to now, this retrieval is not supported by a tool, but through an index schema. However, support for retrieval (e.g., a CBR approach) is considered important for the future.
} 
1. Identify whether the new application to be realised falls into an application class that is covered by an experience module of the cookbook. If this is the case then goto step 2a; else goto step 3 .

2a. Analyse the generic processes, products and methods that are proposed for this application class.

2b. Select the most similar particular application from the specific project level related to this module and analyse the specific description sheets in the context of the current application.

2c. Develop a new project plan and workflow for the new application based on the information selected in steps $2 \mathrm{a}$ and $2 \mathrm{~b}$. Goto step 4 .

3. Develop a new project plan and workflow for the new application by selecting and combining some of the generic processes, products, and methods from the common generic level; make these descriptions more concrete and modify them if necessary.

4. Execute the project by enacting the project plan. Record the experience during the enactment of this project.

5. Decide whether the new project contains new valuable information that should be stored in the experience base. If this is the case, goto step 6, else stop.

6. Document the project using the specific description sheets and enter them into the specific project level of the experience base (supported by the methodology tool).

7. If possible, create a new experience module by generalising the particular application (together with other similar applications) to an application class and generalise the specific descriptions into generic descriptions. Add the new ones to the current cookbook (supported by the methodology tool).

8. If new generic processes, methods, or products could be identified that are of a more general interest, i.e., relevant for more than the application class identified in step 7 , then add them to the common generic level (supported by the methodology tool).

\subsection{The Inreca II Methodology deployed for ISO 9000 and Spice}

In the IT industry, there is an increasing demand for quality assurance standards like ISO 9000 and Spice. In general, a relevant argument for applying an international standard, is the possibility for companies to find more customers for their products. The customers can rely at least on the fact that the development and production of the product followed certain rules like in ISO 9001 which has a model for quality assurance in design/development, production, installation, and servicing. Just to make it clear to the reader it should be mentioned that, e.g., Iso 900x does not guarantee the quality of a product. In fact, the product quality is even not directly mentioned.

CBR applications based on the INRECA methodology, will ease to fulfil the requirements of those standards. The major advantage that can be seen when developing a CBR application following the methodology is the systematic documentation in a predefined format of every single process. The INRECA 
methodology tools support the documentation in a user-friendly way. The methodology itself, as well as the tools for support are documented in detail. Moreover, the documentation of the whole development cycle supports the reuse of the experience and knowledge contained in the projects and leads to a more standardized CBR development process for similar applications.

\section{Building the Experience Base}

We built the experience base through a combination of a top-down and a bottom-up procedure. First, we constructed an initial common generic level by bringing together the experience in the INRECA-II consortium on how to build a CBR application in general. We introduced several processes (and related products and methods) which we considered important and for which we were sure that they would play a role in most CBR applications. In parallel, we started documenting specific CBR projects. Nine different industrial projects that were carried out by the different INRECA II partners have been documented. From these nine projects, three different experience modules could be identified:

Product Catalog Search Module. The basic action is a parametric search by a potential client, or a sales person in the presence of a client, in a product-base or catalogue (see example in the Appendix).

Technical Diagnosis. The task is to introduce a CBR system for trouble shooting and diagnosis for some complex technical equipment, typically via a hot-line telephone service.

Land Use. The basic task is to introduce a CBR system for decision support in use of land.

These modules have been constructed by clustering these nine applications into classes of applications that follow a similar development process. The process models for developing the applications within each such class have then been generalised into a cookbook level module. Variations within the development processes for the applications within such a class usually led to either alternative methods for realising certain processes or more abstract descriptions of the process in the cookbook module.

After these three cookbook modules have been developed, the initial generic level of the experience base was adjusted again to reflect the new experience gained by the specific projects and to make it consistent with the cookbook and the specific level. Through this process, the INRECA-II project has achieved an experience base of significant volume, now consisting of more than 1300 sheets [5]. 


\section{References}

1. Althoff, K.-D. \& Wilke, W. (1997). Potential uses of case-based reasoning in the experience-based construction of software systems. In: R. Bergmann \& W. Wilke (eds.), Proceedings of the $5^{\text {th }}$ German Workshop in Case-Based Reasoning (GWCBR'97), LSA-9701E, Centre for Learning Systems and Applications (LSA), University of Kaiserslautern.

2. Basili, Caldiera, \& Rombach (1994). The Experience Factory. In J. Marciniak (Ed.) Encyclopedia of Software Engineering - Vol 1. New York: Wiley.

3. Bergmann, R., Wilke, W., Althoff, K.-D., Breen, S., Johnston, R. (1997). Ingredients for Developing a Case-Based Reasoning Methodology. In: R. Bergmann \& W. Wilke (eds.), Proceedings of the $5^{\text {th }}$ German Workshop in Case-Based Reasoning (GWCBR'97), LSA-9701E, University of Kaiserslautern, pp. 49-58.

4. Bergmann, R., Wilke, W., \& Schumacher, J. (1997b). Using software process modeling for building a case-based reasoning methodology: Basic approach and case study. In: D. Leake \& E. Plaza (eds.) Case-Based Reasoning Research and Development (ICCBR'97). Lecture Notes in AI. Springer, pp. 509-518.

5. Bergmann, R., Breen, S., Göker, M., Johnston, R., Schumacher, J., Stahl, A., Traphöner, R., Wilke, W. (1998). Refined methodology for building and maintaining a CBR application. INRECA-Deliverable D6 and CDROM.

6. Rombach \& Verlage (1995). Directions in Software Process Research. Advances in Computers, Vol. 41, Academic Press. 


\section{Appendix: Example Sheet ${ }^{3}$}

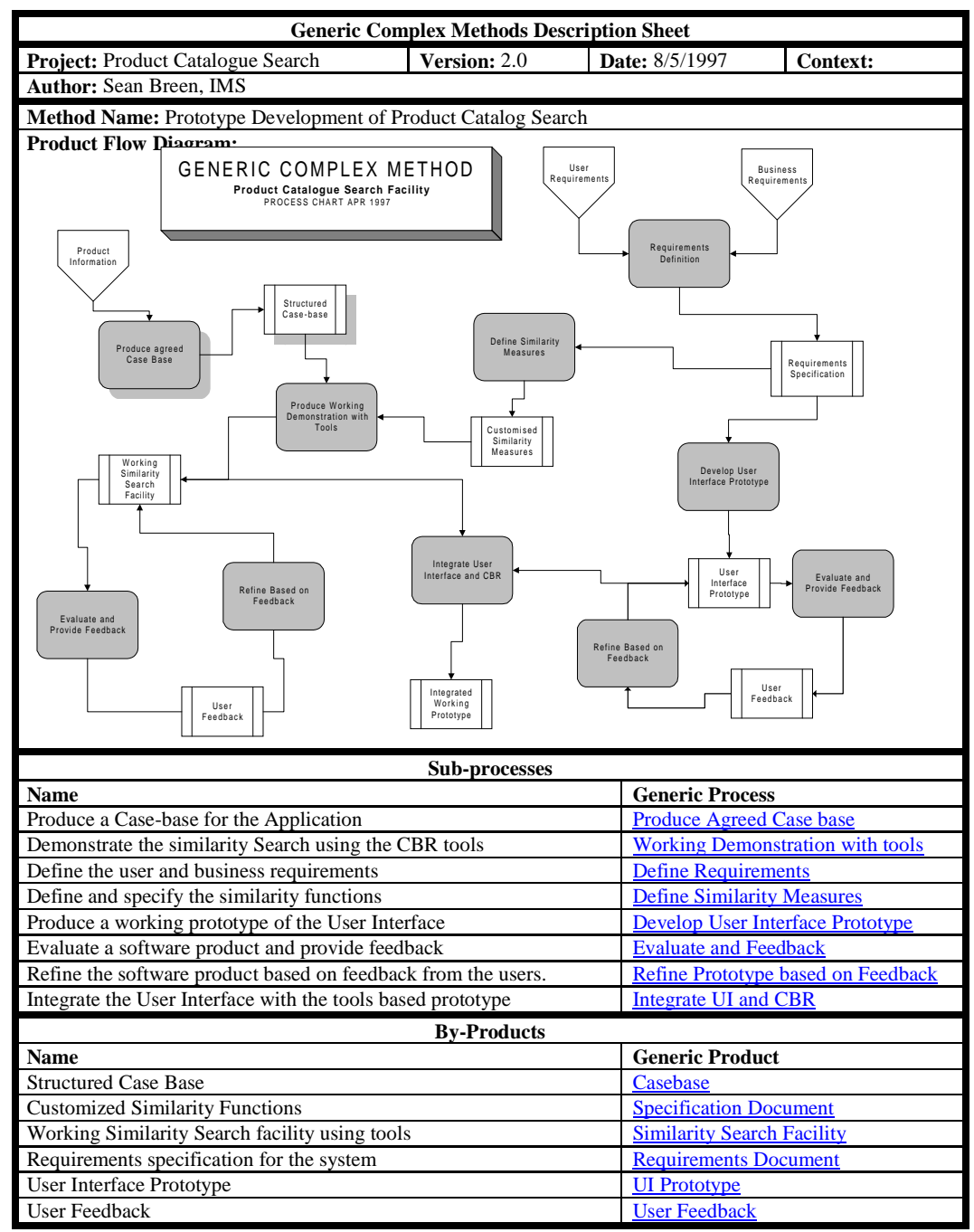

${ }^{3}$ This example sheet was produced by Interactive Multimedia Systems (IMS), Dublin. 